\title{
RECEIVING ANTENNA ELECTRODYNAMIC MODEL IN TERMS OF WAVEGUIDE REPRESENTATION OF HF FIELD
}

\author{
V.V. Khakhinov \\ Institute of Solar-Terrestrial Physics SB RAS, \\ Irkutsk,Russia, khakhin@iszf.irk.ru
}

\begin{abstract}
The receiving antenna is an important part of a radio channel that requires electrodynamic approach in mathematical simulation of its characteristics. Since the invention of radio, and due to further theoretical studies of radio signal transmission, the following situation has arisen: researchers' attention to receiving antennas is inversely proportional to the factor by which their number exceeds the number of transmitting antennas. We address the problem of building a receiving antenna electrodynamic model in terms of a waveguide representation of HF field. Structurally, the antenna is considered as metal wires of a finite length and arbitrary configuration. Current distribution in antenna is calculated using the long-line theory and normal-mode approach. The mathematical representation of the receiving antenna electrodynamic model is calculation expressions for receiving coefficients of normal modes. They reflect the effects of receiving antenna characteristics,
\end{abstract}

including its directional pattern, on effectiveness of the incident HF field energy conversion into the energy of the driven current waves and final distribution of net current in antenna. These expressions are used to derive the expression to calculate the effective length of the receiving antenna.

The obtained mathematical expressions of the receiving antenna electrodynamic model do not contradict the principle of antenna reciprocity.

We present calculation formulas for the receiving coefficients and excitation of the isotropic antenna electromagnetic model.

Keywords: HF field, receiving antenna, Earth — ionosphere waveguide, normal-mode approach.

\section{INTRODUCTION}

Most problems in designing communication systems are solved by the mathematical simulation method. The simulation is aimed at developing a calculation model of radio channel - the part of the communication channel in which an information signal has a spatial distribution [Khakhinov, Kurkin, 2006]. These models are exhaustively characterized by a transfer function and determine characteristics of the output signal (voltage or current modulation at the output of the receiving antenna) with known characteristics of the input signal (voltage or current modulation at the input of the transmitting antenna). Structurally, the model of radio channel generally consists of a transmitting antenna-feeder system, radio propagation environment, and receiving antennafeeder system. In this case, the transfer function of the radio channel can be represented as a product of transfer functions of each of the components.

In the high-frequency (HF) communication, a component of the radio channel is the Earth-ionosphere waveguide. Antenna-feeder systems are metal wires, the current distribution in which is calculated from the long-line theory [Aizenberg et al., 1985] or from the skin-effect theory [Vainshtein, 1988], using Leontovich approximate boundary conditions [Leontovich, 1985]. Electrodynamic models of transmitting and receiving antennas are defined by the electromagnetic field representation.

Within the waveguide representation of HF radio propagation in the Earth - ionosphere waveguide, a normalmode approach (NMA) was developed [Kurkin et al., 1981] to calculate field characteristics with account for the current distribution in an arbitrary transmitting antenna [Kurkin, Khakhinov, 1984]. The first results of the simulation of a decameter radio channel based on NMA are reported in [Altyntseva et al., 1987]. However, the radio channel of interest did not include a receiving antenna, i.e. a result of this work was an HF radio propagation model analogous to that described in [Ponomarchuk et al., 2014]. Khakhinov [2004] presents a simplified model of radio decameter radio channel with isotropic transmitting and receiving antennas.

It seems to be impossible to use the known theorem of antenna reciprocity [Feinberg, 1961] to find the current value at the output of the receiving antenna. The theorem implies that the directional response of the arbitrary antenna is the same for transmission and reception, but it is impossible to calculate the current distribution in it.

The current distribution in a receiving antenna was first calculated by Khakhinov [2000a], using NMA in electrodynamic formulation of the problem. The first results obtained during the development of the receiving antenna electrodynamic model in the waveguide representation of incident HF field were reported in [Khakhinov, 2002].

\section{FORMULATION OF THE PROBLEM}

In the external HF field there is a receiving antenna made of metal wires of a finite length and arbitrary configuration. It is assumed that the antenna keeps the structure of the external field. A geocentric coordinate system with the polar axis passing through the phase center of the transmitting antenna is used. The current distribution in the transmitting antenna can be expressed 
as a product of two functions: $\mathbf{I}(r)$, which determines the spatial distribution, and $u(t)$, which describes the time dependence and called a signal. Modeling of processes of signal propagation in HF radio channels is an objective of many studies.

The purpose of this work is to construct a receiving antenna electrodynamic model whose mathematical representation will allow us to calculate the current at the receiver end from characteristics of the external HF field.

\section{NORMAL MODE RECEIVING COEFFICIENTS}

The receiving antenna electrodynamic model uses the representation of the external electromagnetic field, which, in turn, is determined by the method of solving the electrodynamic problem of radio propagation. The receiving antenna model has been constructed in terms of the waveguide representation of HF field. For simplicity and clarity, the spherically symmetric model of the Earth - ionosphere waveguide is considered. Field characteristics are calculated using NMA [Bremmer, $\underline{1949}$, Kurkin et al., 1981, Khakhinov, 2000b].

Current is induced by the electric field component along the wire axis $E_{l}$ whose value is taken at points infinitely close to the wire surface [Vainshtein, 1988]. In the element of wire length $d l$, the electric field induces the electromotive force (EMF):

$$
d \varepsilon\left(r_{l}\right)=E\left(r_{l}\right) d l \text {. }
$$

In electrodynamics, the antenna wire is a long uniform line with EMF distributed along it. To calculate the current distribution in the antenna, adopt the travelingwave superposition method [Lavrov, Knyazev, 1965].

In the spherically symmetric waveguide, the incident electromagnetic field decays into a TM-type wave field (marked with «e») comprising the $E_{r}$ and $E_{\theta}$ electric field components and a TE-type wave field (marked with «m») comprising the $E_{\varphi}$ component. The expression for EMF induced in the antenna element $d l$ can be written as:

$$
\begin{aligned}
& d \varepsilon\left(r_{l}\right)=E\left(r_{l}\right) d l=d \varepsilon^{e}\left(r_{l}\right)+d \varepsilon^{m}\left(r_{l}\right)= \\
& =\left[\left(e_{r} e_{l}\right) E_{r}\left(r_{l}\right)+\left(e_{\theta} e_{l}\right) E_{\theta}\left(r_{l}\right)\right] d l+ \\
& +\left(e_{j} e_{l}\right) E_{j}\left(r_{l}\right) d l .
\end{aligned}
$$

Here $\mathbf{e}_{r}, \mathbf{e}_{\theta}, \mathbf{e}_{\varphi}$ are unit vectors of the coordinate system, $\mathbf{r}_{l}=\left(r_{l}, \theta_{l}, \varphi_{l}\right)$ is the radius vector of the receiving antenna element, $\mathbf{e}_{l}$ is the unit vector along $d l$.

In general, the expression for the $j$ th component of the field is a set of normal modes

$$
E_{j}\left(r_{l}\right)=\frac{1}{r_{l}} \sum_{n} A_{n} R_{j n}\left(r_{l}\right) D_{j n}\left(I, j_{l}\right) e^{i\left(v_{n} \theta_{l}-\pi / 4\right)} .
$$

Here, $A_{n}$ is the amplitude factor depending on the selected system of units and transmitter power; $D_{j n}\left(\mathbf{I}, \varphi_{l}\right)$ are normal mode excitation coefficients characterizing the distribution of emitted energy over normal modes of the transmitting antenna with specified current distribution I $(r)$ [Kurkin, Khakhinov, 1984]; $R_{n}$ and $v_{n}$ are eigenfunctions and eigenvalues of the corresponding boundary problems for TM and TE waves.

The induced EMF generates two traveling current waves: from $d l$ to the receiving end of the antenna and to the end of the antenna with load resistance $Z$. At the antenna ends, waves are partially reflected, partially absorbed in the load resistor or go away along the feeder line to the receiver. The current was calculated using the traveling-wave superposition method. Summing up all the components of the traveling and reflected waves, it is possible, according to [Lavrov, Knyazev, 1965], to derive an expression for the current at an arbitrary point of the antenna. The value of the current at the receiving end of the antenna can be written as

$$
d j_{0}=\frac{Y(l)}{W} d \varepsilon .
$$

Here, the function

$$
Y(l)=\frac{e^{i k l}+p_{F} e^{i k l}+p_{Z} e^{i k(2 L-l)}+p_{F} p_{Z} e^{i k(2 L-l)}}{2\left(1-p_{F} p_{Z} e^{i 2 k L}\right)}
$$

determines the current distribution in the antenna in terms of loading conditions at both ends, $W$ is the wave resistance of wire, $k$ is the wavenumber, $p_{\mathrm{F}}=\left(W-Z_{\mathrm{F}}\right) /$ $\left./ W+Z_{\mathrm{F}}\right)$ and $p_{\mathrm{Z}}=(W-Z) /(W+Z)$ are coefficients of current reflection from antenna ends, $Z_{F}$ is the input resistance of the feeder line loaded by a receiver, $L$ is the antenna wire length.

Substitute (1) in (3), using expressions (2) for the field components. The net output current at the antenna end $\mathbf{r}_{\mathrm{F}}=\left(r_{\mathrm{F}}, \theta_{\mathrm{F}}, \varphi_{\mathrm{F}}\right)$, loaded by the feeder line, is determined by integrating over the length of the receiving antenna:

$$
J_{F}=\sum_{n} A_{n}\left(D_{n}^{e} P_{n}^{e} e^{i v_{n}^{e} \theta_{l}}+D_{n}^{m} P_{n}^{m} e^{i v_{n}^{m} \theta_{l}}\right) .
$$

In the integration, the linear antenna size smallness condition is used as compared with the distance to the transmitting antenna. Functions

$$
\begin{aligned}
& P_{n}^{e}=\int_{l} \frac{Y(l)}{W}\left[\left(e_{r} e_{l}\right) \frac{v_{n}}{k r_{l}} R_{n}^{e}\left(r_{l}\right)-\left(e_{\theta} e_{l}\right) \frac{d R_{n}^{e}\left(r_{l}\right)}{i k \varepsilon^{\prime} d r}\right] \times \\
& \times e^{-i v_{n}^{e} \theta_{l} \cos \left(j_{F}-j_{l}\right)} d l, \\
& P_{n}^{m}=\int_{l}\left(e_{j} e_{l}\right) \frac{Y(l)}{W} R_{n}^{m}\left(r_{l}\right) e^{-i v_{n}^{m} \theta_{l} \cos \left(j_{F}-j_{l}\right)} d l
\end{aligned}
$$

characterize the level of induced current by TM- and TE-field components of a separate normal mode and are defined by parameters of the receiving antenna. Therefore, it is logical to call $P_{n}^{e, m}$ receiving coefficients of normal modes with corresponding polarization.

Calculation expressions (5) constitute a mathematical representation of the receiving antenna electrodynamic model in terms of the waveguide representation of HF field. They reflect the influence of characteristics of the receiving antenna, including its directional pattern (DP), on the efficiency of the conversion of the external HF-field energy into the energy of driven current waves and on the final net current distribution in the antenna. Depending on antenna design and boundary conditions at the ends, the current distribution with a node at the receiving end of the antenna can be formed; hence there is no signal in the receiver. The same result can be obtained if the transmitter works from the direction in 
which DP (normal mode receiving coefficients) has a low (close to zero) receive level.

Khakhinov [2000a] shows that the normal mode receiving coefficients correspond to the expressions for normal mode excitation coefficients with arbitrarily specified net current distribution for transmission. Efficiency of excitation and reception of normal modes by one antenna is the same for transmission and reception. This means that the resulting mathematical expressions of the receiving antenna electrodynamic model do not contradict the principle of antenna reciprocity.

It is, however, necessary to construct antenna electrodynamic models separately for receiving and transmitting modes, depending on the task in hand.

\section{EFFECTIVE LENGTH OF RECEIVING ANTENNA}

One of the main characteristics of the receiving antenna is its effective length (height), defined as the ratio of voltage at the receiver input (receiving end of the antenna) to incident wave field strength [Lavrov, Knyazev, 1965]:

$$
h_{d}=\frac{J_{F}}{E\left(r_{F}\right)}\left(Z_{A}+Z_{F}\right),
$$

where $Z_{\mathrm{A}}$ is the antenna resistance in terms of the load resistance $Z$.

If the expression for $Y(l)$ is transformed into $Y(l)=\frac{\tilde{Y}(l)}{Z_{A}+Z_{F}}$, the expression for the current length of the antenna can be written as

$$
h_{d}=\frac{1}{E\left(r_{F}\right)} \sum A_{n}\left(D_{n}^{e} \widetilde{P_{n}^{e}} e^{i v_{n}^{e} \theta_{s}}+D_{n}^{m} \widetilde{P_{n}^{m}} e^{i v_{n}^{m} \theta_{s}}\right),
$$

where $\widetilde{P_{n}^{e}}$ and $\widetilde{P_{n}^{m}}$ are defined by formulas (5) with the substitution of $Y(l)$ for $\tilde{Y}(l)$.

\section{ISOTROPIC ANTENNA MODEL}

In research problems it is often necessary to exclude the influence of directional responses of receiving and/or transmitting antennas, e.g. when studying the effect of geophysical parameters of radio channel on radiophysical characteristics of HF signal. The solution requires a radio channel model with antennas having isotropic DP in transmitting and receiving modes.

In this problem it is sufficient to restrict ourselves to the WKB (Wentzel — Kramers — Brillouin) representation of radial functions [Kurkin et al., 1981]

$$
R_{n}^{e, m}=C_{n}\left[e^{i X_{n}^{e, m}}+e^{-i X_{n}^{e, m}}\right] .
$$

Here $C_{n}$ is the normalizing factor, $X_{n}$ are determined by geophysical properties of the Earth — ionosphere waveguide.

Apply the analysis method used in [Khakhinov, 2000b]. It is based on the approximate Poisson summation formula [Popov, Potekhin, 1982]. Series (2) is converted to the integral that is calculated by the stationary phase method. The resulting calculation formulas are simple, and each value has a known geometrical optics interpretation [Khakhinov, 2000b, 2002].

Separate in the formulas for $D_{n}^{e, m}$ [Kurkin, Khakhinov, 1984] functions responsible for the conversion of the excitation current energy in the transmitting antenna into the HF-field energy and its distribution over normal modes. In the formulas for $P_{n}^{e, m}$, select functions determining the conversion of the incident HF-field energy distributed over normal modes into the current energy in the receiving antenna. These functions depend on electrical parameters and geometry of antennas and waveguide (electrical properties of the underlying surface). A condition of antenna isotropy is the equality of these functions to a constant value (unity). Then, the standardized coefficients of excitation and reception of normal modes for isotropic antennas have the form

$$
\begin{aligned}
& D_{n}(I)=I \frac{C_{n}}{k r_{I}} e^{-i X_{n}\left(r_{I}\right)}, \\
& P_{n}=\frac{1}{W} \frac{C_{n}}{k r_{F}} e^{-i X_{n}\left(r_{F}\right)} .
\end{aligned}
$$

Here $r_{I}$ is the radial coordinate of the phase center of the transmitting antenna.

\section{CONCLUSION}

A receiving antenna electrodynamic model has been constructed using the normal-mode approach. The model is mathematically represented by formulas (5) to calculate the normal mode coefficients $P_{n}^{e}$ and $P_{n}^{m}$. They reflect the influence of characteristics of the receiving antenna - primarily its directional response, when the energy of TE and TM waves of incident electromagnetic field is converted into the energy of current oscillations.

The receiving antenna electrodynamic model enabled us to complete the construction of the ionospheric radio channel model [Khakhinov, Kurkin, 2006] and derive a general expression for the transfer function. This model was utilized to analyze the results of oblique sounding, obtained from the ISTP SB RAS network of chirp ionosondes.

The derived formulas for coefficients of excitation and reception of normal modes for wire antennas of virtually all types used in the HF band, are entered into the hardware and software complex of diagnostics and forecast of the ionosphere, plasmasphere, and HF radio channel. This complex has been developed at ISTP SB RAS and is widely used in basic and applied research.

A program has been designed and registered [Kurkin et al., 2017], in which normal mode characteristics are calculated in terms of the properties used by the transmitting antenna. We plan to register a program in which amplitude characteristics of HF signals are computed using the normal-mode approach in view of projected or used transceiver antenna-feeder systems.

The work was performed with budgetary funding of Basic Research program II.12. The results were obtained using the equipment of Center for Common Use “Angara” http://ckp-rf.ru/ckp/3056. 


\section{REFERENCES}

Aizenberg G.Z., Belousov S.P., Zhurbenko E.M., Kliger G.A., Kurashov A.G. Korotkovolnovye antenny [Shortwave antennas]. M .: Radio i Svyaz Publ. 1985, 536 p. (In Russian).

Altyntseva V.I., Ilyin N.V., Kurkin V.I., Orlov A.I, Orlov I.I., Polekh N.M., Ponomarchuk S.N., Khakhinov V.V. Modeling a decameter radiochannel based on normal-mode approach. Tekhnika sredstv svyazi [Technology of communications assets] Ser. SS. Moscow, Ekos Publ., 1987, iss. 5, pp. 28-34. (In Russian).

Bremmer H. Terrestrial Radio Waves. Theory of Propagation. Amsterdam, 1949, 343 p.

Feinberg E.L. Rasprostranenie radiovoln vdol zemnoi poverkhnosti [Propagation of radiowaves along the terrestrial surface]. Moscow, USSR Academy of Sciences Publ., 1961, 548 p. (In Russian).

Khakhinov V.V. Calculation of current in receiving antenna in HF field generated by a series of normal modes. Issledovaniya po geomagnetizmu, aeronomii I fizike Solntsa [Res. on Geomagnetism, Aeronomy and Solar Physics]. 2000a, iss. 111, pp. 74-83. (In Russian).

Khakhinov V.V. Analyzing the HF field in the wave zone of the antenna using the normal-mode approach. Proc. VIII International Conference on Mathematical Methods in Electromagnetic Theory. IEEE: Kharkov, Ukraine, 2000b, pp. 298-300.

Khakhinov V.V. Electromagnetic model of the receiving antenna in terms of a waveguide representation of the HF field. Proc. IX International Conference on Mathematical Methods in Electromagnetic Theory. IEEE: Kiev, Ukraine, 2002, vol. 2, pp. 617-619.

Khakhinov V.V. The electrodynamical model of decameter radiochannel with isotropic receiving-transmitting antennas. Proc. X International Conference on Mathematical Methods in Electromagnetic Theory. IEEE: 04EX840. Dnepropetrovsk, Ukraine. 2004, pp. 372-374.

Khakhinov V.V., Kurkin V.I. Waveguide approach to modeling of the ionosphere radio channel. Proc. of the XI International Conference on Mathematical Methods in Electromagnetic Theory. IEEE: 06EX1428, Kharkov, Ukraine. 2006, pp. 284-286.
Kurkin V.I., Khakhinov V.V. On excitation of a spherical Earth - ionosphere waveguide using arbitrary current distribution. Issledovaniya po geomagnetizmu, aeronomii I fizike Solntsa [Res. on Geomagnetism, Aeronomy and Solar Physics]. 1984, iss. 69, pp. 16-22. (In Russian).

Kurkin V.I., Orlov I.I., Popov V.N. Metod normalnykh voln $\mathrm{v}$ problem korotkovolnovoi radiosvyazi [Normal-Mode Approach in the Problem of HF Radio Communication]. Moscow, Nauka Publ., 1981, 121 p. (In Russian).

Kurkin V.I., Ilyin N.V., Penzin M.S., Ponomarchuk S.N., Potekhin A.P., Khakhinov V.V. Calculation of characteristics of normal modes in a decameter Earth - ionosphere waveguide. Certificate of Computer Program State Registration No. 2017613880 of 03.04.2017. (In Russian).

Lavrov G.A., Knyazev A.S. Prizemnye i podzemnye antenny [Near-Surface and Subsurface Antennas. Moscow, Nauka Publ., 1965, 472 p. (In Russian).

Leontovich M.A. Izbrannye trudy. Teoreticheskaya Fizika [Selected works. Theoretical Physics]. Moscow, Nauka Publ., 1985, 432 p. (In Russian).

Ponomarchuk S.N., Ilyin N.V., Penzin M.S. The model of radio wave propagation in $1-10 \mathrm{MHz}$ frequency range on the base of normal wave technique. Solnechno-zemnaya fizika [Solar-Terrestrial Physics]. 2014, iss. 25, pp. 33-39. (In Russian).

Popov V.N., Potekhin A.P. Field structure of a pulse decameter signal in the Earth-ionosphere waveguide. Issledovaniya po geomagnetizmu, aeronomii I fizike Solntsa [Res. on Geomagnetism, Aeronomy and Solar Physics]. 1982, iss. 59, pp. 68-76. (In Russian).

Vainshtein L.A. Elektromagnitnye volny [Electromagnetic waves]. Moscow, Radio i Svyaz Publ. 1988, 440 p. (In Russian).

How to cite this article

Khakhinov V.V. Receiving antenna electrodynamic model in terms of waveguide representation of HF field. Solar-Terrestrial Physics. 2018. vol. 4, iss. 3, pp. 92-95. DOI: $10.12737 /$ stp-43201812. 